\title{
Une couverture nationale
}

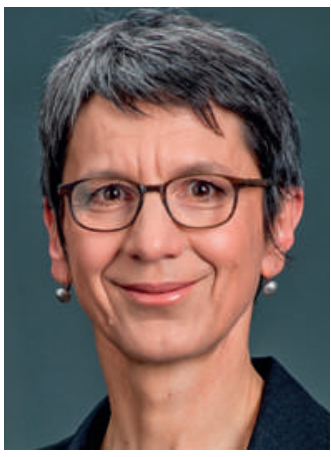

Anna Sax
La Suisse compte trois millions et demi de foyers, qui tous reçoivent un ou plusieurs magazines clientèle de la part de leur assurance-maladie. Ces revues informent sur les derniers produits d'assurance et sur les acteurs de la branche, donnent des recettes et des préconisations pour une vie saine. Moi-même, il m'arrive de feuilleter le magazine de mon assurancemaladie et de m'arrêter sur un article. Cette fois, il s'agit des frais administratifs. Mon assurance-maladie vante l'efficacité du travail des assureurs, comparée à celle des assurances sociales d'Etat. D'après elle, les frais administratifs ne sont nulle part aussi faibles que chez les assurances-maladie sociales. Elle explique que, «contrairement aux entreprises monopolistiques, dans lesquelles les clients n'ont pas de liberté de choix, les assurances-maladie doivent se maintenir sur le marché et proposer le meilleur rapport qualité/prix.» On comprend clairement de quoi treprises privées devant s'affirmer dans un contexte concurrentiel. C'est effectivement le cas pour les assurances complémentaires privées. Mais l'assurance-maladie sociale appartient au domaine public. Le Conseil fédéral déclare en conséquence: «Les informations publiées dans les magazines destinés à la clientèle des assureurs-maladie doivent respecter les principes de l'objectivité, de l'impartialité et de la transparence.» Ces principes ne sont manifestement pas observés par certaines grandes assurances, comptant des centaines de milliers d'assurés, et encore moins par leurs organisations faîtières. Naturellement, il n'est pas simple pour les assurances-maladie de résister à la tentation: elles sont les seules à disposer d'un canal aussi direct pour transmettre leurs messages politiques à la population. Qui ne rêverait pas d'avoir accès aux foyers de tout le territoire suisse pour pouvoir faire passer son message politique? Ces

\section{«La propagande politique ne fait pas partie du cahier des charges d'une assurance-maladie.»}

il s'agit: mieux vaut privilégier une variété d'assurances plutôt qu'une caisse unique. Cependant, l'initiative pour une caisse publique d'assurance-maladie n'est pas mentionnée dans le texte et les assurés ne sont pas invités à voter de façon particulière. Mon assurance-maladie ne fait pas de propagande ouverte avec l'argent de mes primes. Cela me rassure.

D'autres assurances-maladie, nettement plus importantes, ont moins de retenue. Elles font du prosélytisme contre la caisse unique, qu'elles qualifient d'initiative de gauche, et indiquent clairement à leurs assurés, dans le magazine clientèle, comment ils doivent voter le 28 septembre. C'est illégal. Le Conseil fédéral l'a confirmé une nouvelle fois début juin, dans la mesure où «la gestion de l'assurance-maladie sociale est une tâche publique». Cette mission a simplement été confiée aux assureurs, qui doivent donc assumer leur rôle comme le feraient les autorités. En d'autres termes, la propagande politique ne fait pas partie du cahier des charges d'une assurance-maladie. La politique a confié la gestion de l'assurance-maladie sociale aux assureurs. Ces derniers se comportent comme s'ils étaient des en- dernières années, un seul parti politique, dont le chef de file disposait de moyens financiers particulièrement importants, a pu se permettre de faire de la propagande auprès de tous les foyers du pays.

En 2006 déjà, avant la dernière votation sur la caisse unique, un avis de droit reprochait aux assurances-maladie d'utiliser les fonds des primes de façon illicite pour la campagne. Cette fois, les assurances et leur organisation faîtière santésuisse ont fait savoir qu'elles voulaient investir cinq millions de francs dans la campagne contre une caisse publique d'assurance-maladie, tout en déclarant qu'elles n'utiliseraient aucun fonds des assurances-maladie sociales pour la propagande politique. Elles financent leur campagne avec les gains de l'assurance complémentaire privée. Personne ne peut le leur interdire. Mais même en tant que titulaire d'une assurance complémentaire, je ne serais pas ravie à l'idée que l'argent de mes primes contribue au financement d'une campagne politique. 